\title{
Evaluation of urgent caesarian according to colour code at the Befelatanana University Hospital Centre of Obstetric Gynecology in Antananarivo Madagascar
}

\author{
Randriamahavonjy Romuald ${ }^{1}$, Rakotozanany Besaina ${ }^{2}$, Ibrahim Housni ${ }^{2 *}$, \\ Rakotonirina Martial $^{2}$, Rakotoson Koloina Tiphaine ${ }^{3}$, Hery Rakotovao Andrianampanalirivo ${ }^{3}$
}

\author{
${ }^{1}$ Department of Gynecology-Obstetrics, Soavinandriana Hospital Center, Antananarivo, Madagascar \\ ${ }^{2}$ Department of Gynecology-Obstetrics, Befelatanana University Hospital Centre of Obstetric Gynecology in \\ Antananarivo, Madagascar \\ ${ }^{3}$ Departement of Gynecology-Obstetrics, Faculty of Medicine, Antananarivo, Madagascar
}

Received: 10 November 2018

Accepted: 14 May 2019

*Correspondence:

Dr. Ibrahim Housni,

E-mail: lebibavois087@gmail.com

Copyright: ( ) the author(s), publisher and licensee Medip Academy. This is an open-access article distributed under the terms of the Creative Commons Attribution Non-Commercial License, which permits unrestricted non-commercial use, distribution, and reproduction in any medium, provided the original work is properly cited.

\begin{abstract}
Background: Color code was described for the first time in 2003 was described for the first time in 2003 by since and al. The objective of this study was to accomplish urgent caesarian sections according to color code over delays indications-births (DIN) in Motherhood Befelatanana, Antananarivo, Madagascar.

Methods: It was about a longitudinal prospective, analytical study of caesarian sections performed in emergency in CHUGOB going from June $1^{\text {st }}$ till December $31^{\text {st }}, 2017$.

Results: Authors took a census 193 caesarian sections of emergency. Among these patients $28(14.50 \%)$ had a caesarian section encode red, $42(21.76 \%)$ an orange code and $123(63.73 \%)$ a green code. The medium age of the patients was of 26.4 years and that of the gestation was of 37SA and 6 days. The delay indication-birth (DIN) medium was of 102.9 minutes for red code, 99.7 minutes for orange code and 75 minutes for green code.

Conclusions: Authors could not attain DIN of 30 minutes shape in international recommendations. Authors must improve the delay indication entered in the surgical unit by reinforcing knowledge of the agents of support on the management of emergency obstetrical. The possibility of leading to a very quick birth is an indisputable progress in obstetrics but she should not make forget risks inherent in such procedure.
\end{abstract}

Keywords: Caesarian section, Delay indication birth, Emergency, Forecast

\section{INTRODUCTION}

Caesarian section is a surgical operation which allows the extraction of the fetus except the maternal uterus after incision of this one, habitually by way, first abdominal transperitoneal or more seldom retroperitoneal, unusually by way vaginal. ${ }^{1}$ A caesarian section is said urgent, when the vital forecast of the mother and/or of the fetus are immediately included or practically immédiatement. ${ }^{2}$
Some authors offered to classify the indications of caesarian section according to their urgent degree in the course of job. Initially, Lucas and al, in 2000 offered a classification in three groups; not urgent and very urgent, urgent caesarian sections. Then codes colors were introduced to have an optimum management of emergency thanks to a better communication, to reduce the delay extraction-born decision (DDN) and to improve maternal and neonatal forecast. 
He was described for the first time in 2003 by Dupuis and al. Offered DDN is of less than one hour for caesarian sections encode green, less than 30 minutes for caesarian sections encode orange and less than 15 minutes for caesarian sections encode 4 red. $^{3}$

In motherhood as our, the organization in places and within teams does not allow systematically the realization of the procedures of emergency for the recommended delays. The introduction of a coding of caesarian sections in emergency could reduce delays decisions-births. Procedure encodes color, specifying the role of every dealer: gynecologist-obstetrician, anesthetist, commits, pediatrician, midwife, male nurses-anesthetists, male nurses of block and the stretcher-bearers.

The objective of this study was to accomplish the Caesarian sections of emergency according to color code over delays indication-birth (DIN) in Motherhood Befelatanana, Antananarivo-Madagascar.

\section{METHODS}

It is about an analytical longitudinal prospective study of Caesarian sections performed immediately to the Teaching hospital of gynecology-obstetrics Befelatanana (CHUGOB), Antananarivo, Madagascar for a period of six months going from June $1^{\text {st }}$ till December $31^{\text {st }}, 2017$.

All patients having had a caesarian section immediately that it is in the course of job or except job during the period of this study. Programmed caesarian sections become urgent were excluded in this study and the having cesarean section patients immediately but the form was not well filled.

The color used code that is described by Dupuis $\mathrm{O}$ in 2003. Red code is announced when birth is necessary as soon as possible in case of the cord prolapse, distrust of uterine break, bradycardia extended more than ten minutes, strict maternal hemorrhage on afterbirth prævia and the eclampsia. Orange code was suggested for cases anomalies of heart rate foetal persistent. Green code was used in case of failure of progress, in case of dystocic presentation, patient in spontaneous job with prophylactic Caesarian section envisaged later.

The variables of this study were classified in five categories.

- Obstetrical parameters include gestational age, cervical widening and fetal presentation during the decision of indication.

- Maternal parameters include maternal age, parity, antecedents of caesarian section, mortality post caesarian section and post-surgical complications.

- Neonatal parameters include Apgar in the fifth minute, neonatal weight, neonatal transfer and neonatal mortality.
- The mode of anesthesia includes general anesthesia and rachis anesthesia.

- The delay indication birth in minutes include instant of indication, complete team, arrived at the block, instant of the incision and instant of birth.

\section{Inclusion criteria}

- The study was conducted over a period of 2 years from July 2012 to 2014. Gestational age was determined by best estimate from last menstrual period, clinical examination and if ultrasound for fetal biometry in early pregnancy was available

- $\quad$ Preeclampsia was taken as blood pressure $\geq 140 / 90$ $\mathrm{mmHg}$ or more on two occasion at least 6 hours apart with proteinuria as per international society for the study of hypertension in pregnancy.

\section{Exclusion criteria}

- Presence of intrauterine growth restriction (IUGR) was taken as estimated fetal weight on USG less than $10^{\text {th }}$ percentile for gestational age

- Doppler velocimetry was done using Doppler ultrasound Toshiba model (Xario) with $3.5 \mathrm{MHz}$ curvilinear probe. An average of 3 consecutive Doppler velocity waveforms was used for statistical analysis.

\section{Statistical analysis}

Data were collected from a chip of pre-established inquiry and from register of statistics. Recording and data processing were performed from Microsoft Office (Word and Excel) and Ear - information software.

\section{RESULTS}

In the course of studied period, there were 3039 deliveries among which 829 by caesarian section programmed $(27.3 \%)$ and 193 urgent caesarian sections. Among these patients, $28(14.50 \%)$ had a caesarian section encode red, $42(21.76 \%)$ an orange code and 123 $(63.73 \%)$ a green code.

Table 1 represents maternal characters. The age of the population of study was included between 14 years and 45 years with a medium age of 24.44 years. The patients of 29 or more years old were the most doing a cesarean section in emergency in 3 groups of color code. Minimal parity was of zero while the maximum was thirteen averages of which was 0.94 . In 3 groups of color code, the null parity predominated in $48.7 \%$. Twenty-seven $(13.98 \%)$ caesarian sections were associate in a scar uterus, with a more important proportion in groups encode green $(54.1 \%)$ and orange $(29.7 \%)$ which in the group encode red (16.4\%). No maternal decease and postsurgical complication were recorded during this study. 
Table 2 represents obstetrical characters. The mean of gestational age was of thirty-seven weeks of amenorrhea more or less six days with a variance from twenty-three to forty-five weeks of amenorrhea. The gestational age being between thirty-seven-forty-one predominated in
$68.9 \%$. The majority of the fetuses support in cephalic presentation $(96.3 \%)$ and other presentations occupied $3.7 \%$ cases. Concerning cervical widening, more than $41.9 \%$ parturient had a widening between zero for four and $19.8 \%$ in complete widening.

Table 1: Sharing out of the patients according to maternal characteristics and color code of the caesarian sections of emergency.

\begin{tabular}{|c|c|c|c|c|c|}
\hline Variable & & $\operatorname{Red}(n=28)$ & Orange ( $n=42)$ & Green $(n=123)$ & $\%$ \\
\hline \multirow{4}{*}{ Age } & 19 & 6 & 4 & 18 & 14.5 \\
\hline & $19-24$ & 3 & 9 & 36 & 24.9 \\
\hline & $24-29$ & 8 & 10 & 28 & 23.8 \\
\hline & $\geq 29$ & 11 & 19 & 41 & 36.8 \\
\hline \multirow{4}{*}{ Parity } & 0 & 9 & 20 & 65 & 48.7 \\
\hline & 1 & 8 & 8 & 34 & 26.9 \\
\hline & 2 & 5 & 5 & 17 & 14.6 \\
\hline & $\geq 3$ & 6 & 6 & 7 & 9.8 \\
\hline \multirow{2}{*}{ Number of CPN } & $<4$ & 16 & 13 & 48 & 39.8 \\
\hline & $\geq 4$ & 12 & 29 & 75 & 60.2 \\
\hline \multirow{2}{*}{ Antecedent } & HTA ou asthma & 2 & 5 & 3 & 27.1 \\
\hline & Caesarian & 4 & 6 & 17 & 72.9 \\
\hline
\end{tabular}

Table 2: Sharing out of the patients according to characteristics obstetrical and the color code of the caesarian sections of emergency.

\begin{tabular}{|llllll|}
\hline Variable & & Red & Orange & Green & $\%$ \\
\hline \multirow{5}{*}{ Gestational age } & $22-28$ & 3 & 0 & 0 & 1.5 \\
& $28-34$ & 6 & 2 & 8 & 8.3 \\
& $34-37$ & 4 & 6 & 9 & 9.8 \\
\hline \multirow{2}{*}{ Presentation } & $37-41$ & 14 & 31 & 88 & 68.9 \\
\hline \multirow{3}{*}{ Cervical widening } & $\geq 41$ & 1 & 3 & 18 & 11.5 \\
& Cephalic & 28 & 41 & 117 & 96.3 \\
\cline { 2 - 6 } & Not cephalic & 0 & 1 & 6 & 3.7 \\
& $0-4$ & 11 & 19 & 51 & 41.9 \\
\hline & $5-9$ & 13 & 16 & 45 & 38.3 \\
\hline
\end{tabular}

Table 3: Sharing out of the patients according to characters of newborn babies and the color code of the caesarian sections of emergency.

\begin{tabular}{|c|c|c|c|c|c|}
\hline Variables & & Red & Orange & Green & $\%$ \\
\hline \multirow{5}{*}{ Born weight } & $<1500$ & 7 & 1 & 1 & 4.6 \\
\hline & $1500-2000$ & 4 & 5 & 6 & 7.8 \\
\hline & $2000-2500$ & 3 & 5 & 18 & 13.5 \\
\hline & $2500-3000$ & 6 & 14 & 33 & 27.4 \\
\hline & $\geq 3000$ & 8 & 17 & 65 & 46.7 \\
\hline \multirow{2}{*}{ Apgar in the $5^{\text {th }} \mathrm{mn}$} & $<7$ & 16 & 10 & 19 & \\
\hline & $\geq 7$ & 12 & 32 & 104 & \\
\hline \multirow{3}{*}{ Admission there neonatology } & Yes & 21 & 32 & 72 & \\
\hline & No & 7 & 10 & 51 & \\
\hline & $\%$ & 14.5 & 21.8 & 63.7 & \\
\hline \multirow{3}{*}{ Prenatal death } & Yes & 21 & 16 & 33 & \multirow{3}{*}{$\mathrm{p}=0.01$} \\
\hline & No & 7 & 26 & 90 & \\
\hline & $\%$ & 14.5 & 21.8 & 63.7 & \\
\hline
\end{tabular}


Table 4: Sharing out of the patients according to the delay of indication birth (DIN).

\begin{tabular}{|llllll|}
\hline Variables & & Red & Orange & Green & $\%$ \\
\hline Moment of the indication & Before work & 13 & 9 & 3 & 12.7 \\
\cline { 2 - 6 } & During work & 15 & 33 & 120 & 87.3 \\
\hline \multirow{3}{*}{ Time between arrival of drugs and arrival at the block } & $<20$ & 17 & 17 & 60 & 48.7 \\
\cline { 2 - 6 } & $20-30$ & 9 & 8 & 32 & 25.4 \\
\cline { 2 - 6 } Full team constitution delay & $\geq 35$ & 2 & 17 & 31 & 25.9 \\
\hline \multirow{3}{*}{ Time between arrival at the block and incision } & $<60$ & 8 & 12 & 32 & 26.9 \\
\hline & $60-75$ & 5 & 7 & 27 & 20.2 \\
\hline \multirow{3}{*}{ Time between indication and birth } & $75-90$ & 3 & 9 & 15 & 13.9 \\
\hline & $\geq 90$ & 12 & 14 & 49 & 39 \\
\hline & $<20$ & 14 & 19 & 40 & 37.8 \\
\hline & $20-30$ & 6 & 14 & 33 & 27.5 \\
\hline & 230 & 8 & 9 & 50 & 34.7 \\
\hline
\end{tabular}

Table 5: Sharing out of the newborn babies according to delay indication birth and the neonatal admission for green code.

\begin{tabular}{|llll|}
\hline Birth indication time in minutes & Yes $(\mathbf{N}=72)$ & Rate $(\%)$ & $\mathbf{P}$ \\
\hline$<35$ & 0 & 0 & 0.6 \\
\hline $35-50$ & 4 & 5.5 & 0.6 \\
\hline $50-65$ & 4 & 5.5 & 0.9 \\
\hline $65-80$ & 10 & 14 & 0.09 \\
\hline$\geq 80$ & 54 & 75 & 0.01 \\
\hline
\end{tabular}

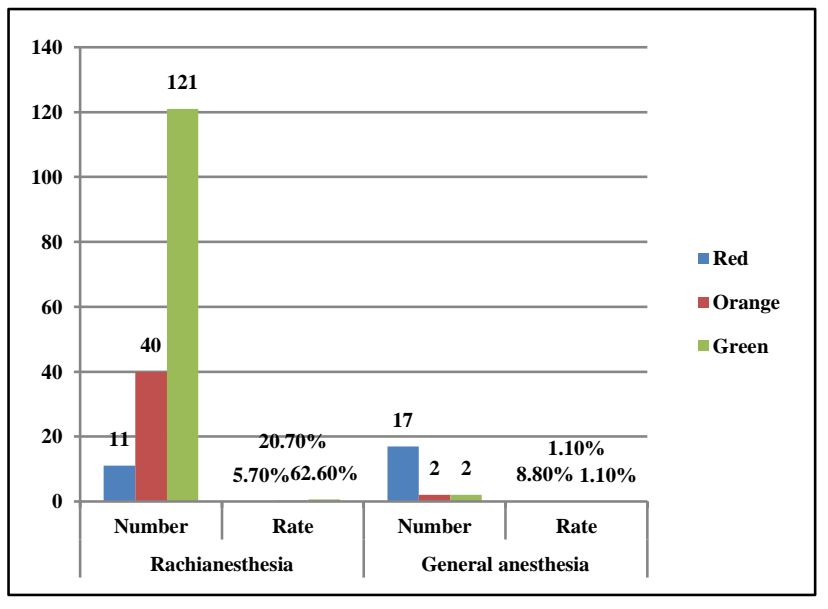

Figure 1: Sharing out of the patients according to the type of anesthesia and the color code of the caesarian sections of emergency.

In this study, author also studied neonatal characters. The Table 3 shows the parameters of the newborn baby. The indication of Apgar in the fifth was included between zero in ten for an average of 8.6. The newborn babies of the green and orange group had a score the upper or equal to seven in the majority of cases, respectively $53.9 \%$ and $16.6 \%$, but for the red group score of less than seven predominated in $83 \%$. Weight minimum was of $200 \mathrm{~g}$ and weight maximum was of $5000 \mathrm{~g}$ with a means of $2826.7 \mathrm{~g}$. The weighty edge enters $2100 \mathrm{~g}$ in $3000 \mathrm{~g}$ occupied most cases (27.4\%). Concerning admission in neonatal intensive care, more half of the red, orange group were accepted in neonatal intensive care respectively of $10.9 \%$ and $16.6 \%$. And of $37.3 \%$ cases for the group. When the delay between the indication of urgent caesarian section and the birth of the newborn baby exceeded eighty minutes, the admission in neonatal intensive care was significant for the patients of the green group. Contagious risk was the motive of transfer in neonatal intensive care most often found $(40 \%)$ followed by the meconium $(27.2 \%)$ inhalation and by the prematurity (16.8\%). We deplored 70 neonatal deceases is $36.3 \%$ cases. Reasons of deceases were the following: the neonatal asphyxia, the respiratory distress syndrome, neonatal infection and fetal death in utero and prematurity. For the red group this rate was $10.9 \%, 8.3 \%$ for the orange group and $17.1 \%$ for the green group. The per natal decease for the orange group was significant for the upper delay in eighty-five minutes. In this group $38.1 \%$ of newborn babies die for a length indication birth of more than eighty-five minutes. For the 
green group, one delay indication birth superior in eightyfive minutes, per natal decease was significant. Per natal decease was $26.8 \%$ when the delay indication and birth exceeds eighty-five minutes. Reasons of decease per natal were numerous to know the neonatal asphyxia, the respiratory distress syndrome, prematurity and fetal in utero death. However, the neonatal infection which prevailed in the most part of cases.

His Figure 1 takes back the used mode of anesthesia. More half $(89.1 \%)$ caesarian sections had made under rachi anesthesia and of $11 \%$ under general anesthesia.

Table 4 shows parameters over indication-born delay. The maternal indications, adnexal and fetal were classified according to codes colors respective. Green code dominated $63.6 \%$ on the contrary red code was only in $14.6 \%$ and only orange code was only $21.8 \%$. The big part of the urgent caesarian sections of our study $(87.6 \%)$ was performed during job while $12.7 \%$ were done before work. The delay between the arrival of medicaments and the arrival to the surgical unit was of one minute at the very least and of three hundred and sixty-five minutes at the farthest with an average of 29.54 minutes and a value $\mathrm{p}=0.001$. The delay less than twenty minutes predominated in $48.7 \%$ to arrive at the surgical unit. For the delay of constitution of a complete team, ten minutes the time was minimum to constitute a complete team while four hundred and fifteen minutes was the time maximum with an average of 89.86 minutes, $p=0.004$. The delay of constitution of a complete team was of ninety minutes or more in mostly $(39 \%)$. For delay between the arrival to the block and incision there times, time minimum was of three minutes and the time maximum was of ninety-two minutes with an average of 24.3 minutes (NS). The delay between the arrival to the surgical unit and the incision was less in twenty minutes in the majority of cases $(37.8 \%)$. For delay between incision and birth in minutes, one minute was the time minimum and thirty-five for the maximum with an average of 6.3 minutes (NS). The edge an hour from five till ten minutes occupied the most part of cases. For delay between indication of caesarian section and birth in minutes, the delay minimum was of twenty-five minutes and the delay maximum was of four hundred and twentyfive minutes with an average of 102.8 minutes for red code, 104.4 minutes for orange code and 108.9 minutes for green code. The delay between the indication of caesarian section and the birth of the baby was more than eighty minutes in the majority of cases $(66.3 \%)$.

\section{DISCUSSION}

This study revealed a rate of caesarian section immediately of $6.4 \%$ groups of deliveries and $18.88 \%$ caesarian sections. This rate is high by comparison with data published by other teams. Four studies published between 2002 and 2006 bring back rates of urgent caesarian sections varying deliveries between from 0.4 to $0.7 \%$ groups of and from 2.5 to $3.4 \%$ caesarian sections.
Two successive studies accomplished in 2003 and 2007 in a motherhood of type 3 showed a rate of urgent caesarian sections varying deliveries from 1 to $0.8 \%$ groups of and from 5.5 to $3.4 \%$ complete caesarian sections. ${ }^{5}$ However, the place occupied by caesarian sections accomplished in our centre is much less important than that brought back by other African authors. In the hospital complex of Yaoundé, Nkwabong and al note a higher rate of urgent caesarian sections, $81.9 \%$ between 2000 and $2004 .^{6}$

The relating modesty of our rate of urgent caesarian section explains due to the fact that the Teaching hospital of Gynecology Obstetrics Befelatanana (CHUGOB) is the only centre of public reference in the city of Antananarivo. The majority of the parturient were consulted in service, some people of her did not make regular prenatal consultations. However some cases should have been programmed.

The problems of emergency are in the middle of the concerns of the obstetricians. Recognize indeed needs for II us to know indeed urgent situations and to bring them an adequate answer as soon as possible, without falling in an excessive interventionism. Color codes were introduced to diminish maternal and neonatal morbimortality. ${ }^{7,8}$ The impact of the introduction of color code within motherhood in Madagascar had not been studied, in our knowledge yet.

The total medium age was of 26.4 years with extremes from fourteen to forty-five years. Caesarian section is pointed out at the more 19-years-old women's for 3 groups (red, orange or green codes). The medium age for the red group was of 27.8 years, 28.2 years old for the orange group and of 25.5 years for the green group.

A study accomplished to the teaching hospital Lille (France) between January, 2015 and April, 2017 showed a total medium age of 31 years. The medium age for the red group was of 32 years, 31 years old for the orange group and of 30 years for the green group. ${ }^{3}$ However, a study accomplished in of teaching hospital of Brazzaville found a mean of age of exhibition in urgent caesarian section (everything merged code) similar to our study. There was of 26.3 years with extremes of ages from fourteen to forty-five years. ${ }^{9}$

In France the rate of caesarian section believes directly with age. It is weaker for least than 20 years that is $12.3 \%$ and attains $27.6 \%$ for the forty-year-old women more. ${ }^{10}$ This context can explain due to the fact that in most developing countries, the girls get married in precocious age. This to lighten the load of their parent to the weakwilled persons comes back. This precocious marriage would be at the origin of an unwanted pregnancy or at risk which can train a mechanical dystocia in the course of job. Where from this high rate of caesarian section in developing countries. Concerning parity $48.7 \%$ women was nulliparous. There had nulliparous more having had 
a green code in comparison with other color codes and is also for the scar uteruses there. The study of DeltombeBodart to the teaching hospital of Lille showed that the majority of the having a Cesarean section women support primiparous and having had an orange code in comparison with other color codes (the same official report for scar uterus on color code). ${ }^{3}$ On the contrary a study of the hospital of Hong Kong found that $72 \%$ of having a cesarean section was nulliparous with a predominance of red code. ${ }^{11}$

Several studies showed that most having cesarean section women immediately support with nulliparous, 6.12 what joins the results of our study. ${ }^{4}$ The rate of Caesarian section diminishes therefore with the number of parity and nulliparous would be more subject to the apprehension of delivery. This augments the rate of caesarian section in the course of job by an insufficiency of increase, they were predisposed to anomalies of uterine activity, to cervical anomalies and those of the soft part especially since the basin has never had shown.

In this study, the gestational total medium age was of 37 weeks of amenorrhea (ITS) and 6 days. In $19.6 \%$ cases caesarian sections were performed before $37 \mathrm{HER}$ of amenorrhea which $1.5 \%$ were accomplished before 28 HER for red code.

The age medium gestational was 34 HER for the red code, of 38 HER for orange code and of 38 HER +4 days for green code. Concerning the gestational total mean of age, our result is similar to that of Block in France and to that from Maneschi to Italy. ${ }^{5,13}$ On the contrary our result is less than that of Lecerf who found an age total medium gestational of 36.8 HER. However, most studies in literature found an age total medium gestational $\geq 38$ HER. $^{4,14-20}$

In literature the mean of gestational age for red code was included between 37SA in 40SA, what is widely superior to the results of our study. ${ }^{13,17,20}$ For orange code Sayegh and al found a result similar to this study. ${ }^{20}$ On the contrary Huissoud, Lurie and Maneschi found in their respective studies a mean of gestational age of 39SA for orange code. ${ }^{13,17,19}$ For green code Lurie in Israel found an age medium gestational of 39.4 HER and Maneschi in Italy found a gestational age of 37 HER. ${ }^{13,19}$ These two results differ from ours. The gestational mean of age for the red code of our study was widely low in comparison with that found in literature. This could explain due to the fact that near the women done a cesarean section in our study, their pregnancies were badly followed.

During our study medium total born weight was of $2826.7 \mathrm{~g}$. The medium born weight for red code was of $2025 \mathrm{~g}$, of $2667.7 \mathrm{~g}$ for orange code and of $2981.6 \mathrm{~g}$ for green code. In our study medium total born weight was less than what is found in literature. He varied between $2992.6 \mathrm{~g}$ in 3175 g. $^{14,15,19}$
Concerning the mean of born weight of color codes, our results are widely less of those published in several studies. He varied of $2784.6 \mathrm{~g}$ in $3319.6 \mathrm{~g}$ for red code and of $2979 \mathrm{~g}$ in $3285 \mathrm{~g}$ for orange code. ${ }^{17-21}$ These different results especially for red code could be explained due to the fact that it was urgent premature or born babies that introduced a delay of growth in utero (RCIU) at a hypertense mother. This could lead to a caesarian section of red code. It is not possible to disclaim him also that the malnutrition at the pregnant women as talks about the fetal hypotrophy because most these women come from disadvantaged background.

The score of APGAR is a means allowing assessing the state of the newborn babies at birth. The score of mean APGAR in the fifth minute was. ${ }^{8,6}$ The newborn babies of orange and green code had a score of APGAR $\geq 7-5$ minutes of life. On the contrary the new born of red code had a score of APGAR $<7-5$ minutes of life. Concerning the score of medium APGAR in the fifth minute, our results are similar to those of the Cola trees in Norway, of Sayegh in the Motherhood of the Cross - Russet from Lyons to France and of Onah in Nigeria. ${ }^{18,20,22}$ On the contrary Lurie in Israel, Huissoud in France and Hillemanns in Germany found in their studies a score of lower medium APGAR in our result in 5 minutes of life. This score varied from 2 to 7 .

In the university hospital Femme-Mère-Enfant of Bron Bloc found a score of APGAR $<7-5$ minutes of life for red code what corresponds to the result of our study. ${ }^{5}$ However studies accomplished in Italy by Maneschi and by Sayegh in France found a score of APGAR >7-5 minutes of life for red code. ${ }^{13,20}$ Concerning orange code and green code, found results $d$ years literature was similar to our study (score of APGAR >7-5 minutes of life)..$^{9,13,20}$

Although the risk of neonatal asphyxia in urgent caesarian sections could easily be explained by the biggest frequency of indications especially the anomalies of heart rate fetal either bradycardia or feto-pelvic disproportion. This asphyxia could be increased by the more common use immediately (red code) of the general anesthesia, rather than of the rachianesthesia, this compounds with result that we found with a strong proportion of score of APGAR $<7-5$ minutes life for the red code which was accomplished under general anesthesia. $^{23}$

In this study, more half of cases of red code (10.9\%), orange code and of green code $(40.0 \%)$ was transferred in neonatal intensive care. The motive of transfer in intensive care most found was contagious risk in $40 \%$ cases for any codes, merged. Our results are variable in comparison with those found in literature. Sayegh in Lyons (France) in 2004, found 83 newborn babies were accepted in neonatal intensive care for red code and 12 newborn babies for orange code. The prematurity was the 
first reason of transfer of the newborn babies in neonatal intensive care. ${ }^{20}$

In 2012, Larcef in Caen (France) had shown that 19 newborn babies were transferred in neonatal intensive care for red code, 16 for orange code and 21 for green code. The bradycardia was the first motive of admission in neonatal intensive care. ${ }^{21}$ Cola trees had written in his study that 83 newborn babies were transferred in neonatal intensive care for red code and 407 for green code. ${ }^{18}$

Our study contagious risk occupied the most part of transfers in neonatal intensive care, which can explain by made that the indication of urgent caesarian section was mainly dystocia in $60 \%$ cases, proved by the study made to the hospital of Yaoundé which found that fetal contagious risk often follows extended job.6 The admission in neonatal intensive care was not linked to DIN for red and orange codes on the contrary when DIN exceeded eighty minutes the transfer in neonatal intensive care was significant with $\mathrm{p}<0.01$ for green code.

In our study, total per natal decease was $36.3 \%$. Per natal decease was $10.9 \%$ for red code, $8.3 \%$ for orange code and $17.1 \%$ for the green. Reasons of per natal decease most found for any codes of color merged were the neonatal infection in $44.3 \%$ cases. This rate is high by comparison with the publishing by other teams. Four studies published between 2004 and 2014 bring back rates of variable per natal decease from 0.3 to $4.2 \%$ caesarian sections for all encode of color merged. 5,6,14,17,20,24 However a retrospective study led between 2004 and 2009 to the teaching hospital of Caen showed a per natal death rate of $53.4 \%$ for red code, of $42.3 \%$ for orange code and of $42.9 \%$ for the green. ${ }^{21}$

For red code, per natal decease was significant $(p=0.01)$ as soon as the delay indication birth (DIN) exceeded 75 minutes. It was also significant for codes orange $(\mathrm{p}=$ $0.01)$ and green $(\mathrm{p}=0.00003)$ but as soon as DIN exceeded 85 minutes. It was brought back that DIN of more than 75 minutes was necessary before delay is linked to significantly disadvantageous results for the mother and the fetus. ${ }^{25,26}$ A study noticed no significant difference for the caesarian sections of orange code depending on whether DIN was performed in registered 30 minutes or more. Also, the state of the baby after a caesarian section of red code did not differ significantly depending on whether DIN was of fifteen minutes or more to see in 30 minutes. The continuation of DIN therefore did not influence significantly the state of the newborn babies. ${ }^{17}$ Mofolo and al found that the interval of 30 minutes was accomplishable, but noted that he rule of 30 minutes did not seem to improve materno-fœtal forecast or aggravating. ${ }^{27}$

In our study $89.1 \%$ caesarian sections had taken place under rachianesthesia. The caesarian sections of red code had taken place under general anesthesia contrary to the caesarian sections of codes orange and codes green. Our results are similar to literature..$^{3,4,6,15,24}$ Only the study accomplished to the teaching hospital of Brazzaville showed that the majority of urgent caesarian sections had been made under general anesthesia whatever is color code. ${ }^{9}$ It was shown that the general anesthesia allows to reduce DIN in comparison with the locoregional anesthesia. $^{19,28}$ A general anesthesia is the most recommending in case of extremely urgent caesarian section (red code). ${ }^{15,19,29}$ What was case in our study explaining as well as the type of anesthesia performed in our study had no influence on DIN.

The mean length of the transfer to the surgical unit was of 29.54 minutes. The majority of the patients arrived at the block in less than 20 minutes for all encode of color merged $(48.7 \%)$. Our results are widely low in comparison with those find in literature. Linck had shown that length decision arrived at the block varied between 22.2-26.2 minutes. ${ }^{14}$ For Sayegh this length was of 10 minutes in means. ${ }^{20}$ As for Huissoud the length of transfer to the block varied on average between 4.8-11.2 minutes. ${ }^{14}$ This difference could explain in first of made that our centre was the only one center reference of the city of Antananarivo in surgical-obstetrical emergency. Secondly, two of our 3 blocks surgical are arranged on the first floor where from difficulties of transfer of parturient to the block (lift out of repair or not available stretcher-bearer). Many publications suggest that the delay decision arrived at the surgical unit is the main criterion on which DIN. ${ }^{15,20,30}$ The authors showed that $45.6 \%$ medium DIN corresponds in this time of transfer. ${ }^{15,31}$ Other authors showed that when the woman arrived at the surgical unit in 10 minutes according to the decision-making, DIN was quick in $81.8 \%$ cases 15.32 . What explains why this delay was a factor postponing DIN in our study $(\mathrm{p}=0.001)$.

This delay was significant in comparison with DIN $(\mathrm{P}=0.004)$. This rate is widely superior in comparison with other studies. Three studies published between 2004 in 2008 showed that the delay of constitution of a complete team varied between 10 - 18 minutes. He had not influenced DIN. ${ }^{15,20,22}$ In our study, the delay of constitution of a complete team can explains by the lack of personnel. Indeed, the anesthetist and the surgeon can be occupied in the block and the neonatologist preoccupied to manage other pediatric emergency. Where from the influx of the women there attempt an urgent caesarian section.

The total medium delay between the arrival to the surgical unit and the incision was of 24.3 minutes. This delay was of 23.1 minutes for the red code, of 20.1 for orange code and of 25 minutes for green code (Table 4). Our results are widely high by report those found in literature. According to Huissoud and MC Cahon the total medium delay between the arrival to the surgical unit and the incision varied between 3.3 - 7.3 minutes. ${ }^{14,28}$ Dupuis in 2003 showed that this total delay was of 13 minutes in means and of 14.6 minutes for red code. ${ }^{15} \mathrm{~A}$ 
study accomplished in a motherhood of type I showed that the total medium delay between the arrival to the block and the incision was of 18.7 minutes. ${ }^{4}$ Several studies showed that the delay between the arrival to the surgical unit and the incision did not influence DIN. ${ }^{15,25}$

This is similar to present study. In this study, DIN was of 102.9 minutes for red code, of 99.7 minutes for orange code and of 109.5 minutes for green code. Several studies accomplished in motherhood of level III as ours, DIN was less than 30 minutes for red code..$^{3-5,13-17}$ In some studies, this delay exceeded 30 minutes for red code..$^{9,20,33-36}$ We did not succeed in attaining recommendations for every code of color as it is case in several developing countries..$^{20,33-36}$ This could explain by several to know:

- Low-grade women socioeconomic

- Maladjusted in fractures in color codes

- Agents of supports not formed in management of emergency obstetrical.

\section{CONCLUSION}

Authors could not attain DIN of 30 minutes in extremely urgent caesarian section (red code), in accordance with international recommendations. However the introduction of color codes and implementation of the devoted protocols will allow organizing into a hierarchy the urgent degree of caesarian sections and bringing a benefit in the reduction of DIN in our motherhood. A systematization of gestures is to favor to improve these delays notably the delay indication - entered in the surgical unit, for cases of extreme emergency.

However, insurance to be able to intervene fast is not always the guarantor of a neonatal good condition. He is indeed sure that there are cases where it is very necessary to act quickly and what in material, as in many others. You can't make up for lost time; but that you should not either merge speed with haste or act judiciousness. It seems also interesting to lean over new working axles, as an addition of the permanent collection of data with organization of audits and regular multiple-subject meetings to blot to check targets off to be improved considering the introduced files.

Funding: No funding sources Conflict of interest: None declared

Ethical approval: Not required

\section{REFERENCES}

1. Broch DE, Courtois L, Maillet R, Riethmuller D. Caesarean sections. EMC (Elsevier Masson SAS, Paris). Obstetrics. 2008;5:102-A-10.

2. Lucas DN, Yentis SM, Kinsella SM, Holdcroft A, May AE, Wee M, et al. Urgency of cesarean section: a new classification. JR Soc Med. 2000;93:346-50.
3. Deltombe-Bodart S, Grabarz A, Ramdane N, Delporte V, Depret S, Deruelle P, et al. Compliance to the color codes protocol according to the indication of cesarean and to the decision-to-delivery interval. Gynecol Obs Fertil Senol. 2018;46(78):575-9.

4. Linck C, Choserot M, Cristinelli S, Callec R, Morel O. Emergency caesarean sections in primary care maternity: Impact of a color code. J Gynecol Obs Biol Reprod. 2016;45(7):701-7.

5. Bloc F, Dupuis O, Massardier J, Gaucherand P, Doret M. Are we overusing of crash c-section procedure?. J Gynecol Obs Biol Reproduction. 2010;39(2):133-8.

6. Foumane P, Mve Koh V, Ze Minkande J, Njofang Ngantcha EA, Dohbit JS, et al. Risk factors and prognosis for emergency cesarean section at the Gynecology Obstetrics and Pediatric Hospital, Yaounde, Cameroon. Med Sante Trop. 2014;24:8993.

7. Weiner E, Bar J, Fainstein N, Ben-Haroush A, Sadan $\mathrm{O}$, Golan A, et al. The effect of a program to shorten the decision-to-delivery interval for emergent cesarean section on maternal and nonatal outcome. Am J Obstet Gynecol. 2014;210(3):El-224.e6.

8. Figueireido S, Tsatsaris V, Mignon A. Anaesthetic management for acute foetal distress. Ann Fr Anesth Réanim. 2007;26(7):699-704.

9. Mbongo JA, Butoyi JM, Papandi-Ikourou A, Iloki LH. Caesarean section in emergency at the Brazzaville University and Hospital Center. Afr J Med Health Sci. 2016;17(1):2-3.

10. High Authority of Health. Ceasarean planned for the future service evaluation and improvement of practices: HAS; 2014.

11. Chown KM, Mak SL. Maternal and fetal outcomes in extremely urgent caesarean delivery in relation to the decision-todelivery interval. Hong Kong J Gynaecol Obstet Midwifery. 2015;15(1):16-22.

12. Bello FA, Tsele TA, Oluwasola TO. Decision-todelivery intervals and perinatal outcomes following emergency cesarean delivery in a Nigerian tertiary hospital. Int J Gynaecol Obstet. 2015;130:279-83.

13. Maneschi F, Biccirè D, Santangelo G, Perrone S, Scaini A, Cosentino C. Implementation of the fourcategory classification of cesarean section urgency in clinical practice. a prospective study. Gynecol Obs Investigation. 2017;82(4):371-5.

14. Huissoud C, Mesnildot P, Sayegh I, Dupuis O, Clément HJ, Thévenet $\mathrm{S}$, et al. Color-codes implemention shortens the decision-to-delivery interval of emergency C-sections. J JGYN. 2009;38:51-9.

15. Dupuis O, Sayegh I, Decullier E, Dupont C, Clément $\mathrm{HJ}$, Berland $\mathrm{M}$, et al. Red, orange and green Caesarean sections: a new communication tool for on-call obstetricians. J EJOGRB. 2008;140:206-11.

16. Hirani BA, Mchome BL, Mazuguni NS, Mahande MJ. The decision delivery interval in emergency caesarean section and its associated maternal and 
fetal outcomes at a referral hospital in northern Tanzania: a cross-sectional study. BMC Pregnancy and Childbirth. 2017;17:411.

17. Huissoud C, Dupont C, Canoui-Poitrine, Touzet S, Dubernard G, Rudigoz RC. Decision-to-delivery interval for emergency caesareans in the Aurore perinatal network. J EJOGRB. 2010;149:159-64.

18. Kolas T, Hofoss D, OIAN P. Predictions for the decision-to-delivery interval for emergency cesarean sections in Norway. Acta Obs Gynecol. 2006;85:561-6.

19. Lurie S, Sulema V, Kohen-Sacher B, Sadan O, Glezerman M. The decision to delivery interval in emergency and non-urgent cesarean sections. J EJOGRB. 2004;113:182-5.

20. Sayegh I, Dupuis O, Clement HJ, Rudigoz RC. Evaluating the decision-to-delivery interval in emergency caesarean sections. J EJOGRB. 2004;116:28-33.

21. Lecerf M, Vardon D, Morello R, Lamendour N, Dreyfus M. Can we do a cesarean section in less than 30min in unsuitable premises in order to follow the recommendations of the ACOG?. J Gynecol Obs Biol Reproduction. 2013;42(4):393-400.

22. Onah HE, Ibeziako N, Umezulike AC, Effetie ER, Ogbuokir CM. Decision-delivery interval and perinatal outcome in emergency caesarean sections. J Obstet Gynaecol. 2005;25(4):342-6.

23. Livermore LJ, Cochrane RM. Decision to delivery interval: a retrospective study of 1000 emergency cesarean sections in Norway. Acta Obstet Gynecol Scand. 2006;85(5):561-6.

24. Yaich P, Ouattara A, Koffi N, Sanou J, Itéké F. Caesareans in emergency: maternal-fetal prognosis at Cocody hospital in Abidjan. RAMUR. Mai. 2012;17(1):2-5.

25. Cerbinskaite A, Malone S, McDermott J, Loughney AD. Emergency caesarean section: Influences on the Decision-to-Delivery Interval. Hindawi Pub Corp $\mathbf{J}$ Pregnancy. 2011:6.

26. Thomas J, Paranjothy S, James D. National cross sectional survevy to determine whether the decision ton delivery interval is critical in emergency caesarean section. BMJ. 2004;328(744):665.

27. Mofolo N, Cronjé $\mathrm{P}$, Gobodo $\mathrm{M}$, Magano $\mathrm{O}$, Mothiba M, Pierce N, et al. Adherence to the 30minute rule in emergency caesarean deliveries due to foetal distress at Pelonomi Tertiary Hospital, Bloemfontein, South Africa. PULA: Botswana Afr J Studies. 2017;31(2):2-6.
28. McCahon RA, Catling S. Time required for surgical readiness in emergency caesarean section spinal compared with general anesthesia. Int $\mathbf{J}$ Obstet Anesth. 2003;12:178-82.

29. Lagrew DC, Bush MC, McKeown AM, Lagrew NG. Emergent (crash) cesarean delivery: indications and outcomes. Am J Obstet Gynecol. 2006;194:1638-43.

30. Helmy WH, Jolaoso AS, Ifaturoti OO, Afify SA, Jones MH. The decision-to delivery interval for emergency caesarean section: is 30 minutes a realistic target? BJOG. Int J Obstet Gynaecol. 2002; 109:505-8.

31. Pierre F, Rudigoz RC. Emergency caesarean delivery: Is there an ideal decision-to-delivery interval? J Gynecol Obstet Biol Reprod. 2008;37:417.

32. Lafitte AS, Vardon D, Morello R, Lecerf M, Stewart $\mathrm{Z}$, Dreyfus M. Can we reduce the decisio-to-delivery interval in case of emergency cesarean sections by optimizing the premises 'architecture? EMC Gynecol Obstétrics Fertility Sénol. 2017;45:590-5.

33. Tolcher MC, Johnson RL, El-Nashar SA, West CP. Decision-to-Incision Time and neonatal Outcomes. Obstet Gynecol. 2014;123:536-48.

34. Pearson GA, Kelly B, Russell R, Dutton S, Kurinczuk JJ, et al. Target decision to delivery intervals for emergency caesarean section based on neonatal outcomes and three year follow-up. Eur J Obstet Reprod Biol. 2011;159:276-81.

35. Mishra N, Gupta R, Singh N. Decision Delivery Interval in Emergency and Urgent Caesarean Sections: Need to Reconsider the Recommendations?. J Obs Gynecol India. 2018;68(1):20-6..

36. Bello FA, Tsele TA, Oluwasola TO. Decision-todelivery intervals and perinatal outcomes following emergency cesarean delivery in a Niger tertiary hospital. Int J Gynaecol Obstet. 2015;130:279-83.

Cite this article as: Romuald R, Besaina R, Housni I, Martial R, Tiphaine RK, Andrianampanalirivo HR. Evaluation of urgent caesarian according to colour code at the Befelatanana University hospital centre of obstetric gynecology in Antananarivo Madagascar. Int J Reprod Contracept Obstet Gynecol 2019;8:2193-201. 